\title{
The impact of smoking in the home on the health outcomes of non-smoker occupants in the UK
}

\author{
Jeanette Kusel ${ }^{1 *}$, Beth Timm ${ }^{1}$ and lan Lockhart ${ }^{2}$
}

\begin{abstract}
Smoking in the home remains a key source of exposure to secondhand smoke for non-smokers, particularly since the UK public smoking ban in 2007. A systematic literature review was conducted to identify all UK evidence on the impact of secondhand smoke exposure in the home on health and behavioural outcomes in non-smoker occupants. MEDLINE, EMBASE and the Cochrane Library were searched to identify all relevant UK empirical studies from 2000 to June 2011. A qualitative overview of the evidence is presented. Exposure to secondhand smoke in UK homes was found to be associated with serious negative health effects in non-smokers, including significantly increased risk of meningococcal carriage $(p<0.001)$ and disease $(p=0.05)$ in children and adolescents, cognitive impairment $(p<0.001)$ in adults, a higher rate of medically attended accidents in children with smoking mothers $(p<0.01)$, and for non-smoking women, a significant decrease in infant birth weight $(p=0.007)$. Living in a smoking household significantly increased the risk of future regular smoking in children $(p<0.001)$. In conclusion, this systematic review has identified strong evidence of an association between secondhand smoke exposure in the home and several serious health conditions. This finding highlights the importance of educating current smokers on the consequences of non-smoker exposure to smoking in the home.
\end{abstract}

Keywords: Second-hand smoking, Environmental tobacco smoke, Systematic literature review

\section{Introduction}

Secondhand smoke (SHS) is the smoke from the burning tip of a cigarette or exhaled smoke that is then inhaled by non-smokers. On a global scale, SHS was responsible for an estimated 600,000 deaths of non-smokers in 2011, the majority of which were women and children [1]. SHS exposure has been hailed as one of the world's most critical environmental health hazards and there is no reported safe level of exposure [1]. SHS is known to be associated with an increased risk of smoking-related diseases such as lung cancer, heart disease, respiratory infection and meningitis [2-6].

In the UK, $21 \%$ of the population were smoking in 2010 [7]. The smoking ban in the UK has successfully reduced smoking in public places, work places, restaurants and bars, with some evidence of an improvement in health outcomes [8-10]. However, non-smokers living in smoking households continue to be exposed to high

\footnotetext{
*Correspondence: jeanette.kusel@costellomedical.com

${ }^{1}$ Costello Medical Consulting Ltd, St John's Innovation Centre, Cowley Road, Cambridge CB4 OWS, UK

Full list of author information is available at the end of the article
}

levels of SHS in the home and therefore passive exposure remains a major public health issue, particularly for children [11,12]. In $2007,22 \%$ of children aged $4-15$ in the UK lived in a home where someone smoked indoors [12]. Where one parent smoked, this parent did so inside the home in $63 \%$ of cases and where both parents smoked at least one of them did so inside the home in $79 \%$ of cases [12].

The main objective of this review was to collate the current UK evidence on the impact of exposure to SHS in the home on health outcomes of non-smoker occupants. Within this main objective, the review was divided into two distinct areas with the aim of assessing: (1) health outcomes of non-smokers previously and currently exposed to SHS in the home; and (2) risk of current or future smoking in children exposed to second hand smoke in the home.

\section{Methods}

Systematic review methods

A systematic and comprehensive literature search was conducted to identify all empirical studies that considered adverse events associated with exposure to SHS in the home,

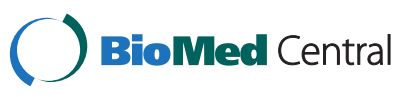


including parental, carer and family smoking in the UK setting. Studies reporting on home smoking were obtained through text word searches relating to 'house,' 'home' or 'domestic', and combined with the MeSH headings 'Tobacco Smoke Pollution', 'Smoking,' 'Smoking Cessation' and 'Tobacco Use Cessation' and relevant text word searches, including search terms for smoking related toxins/particles. Using these search strategies, the MEDLINE (including MEDLINE In Process), EMBASE and Cochrane Library databases were searched to June 2011. Search results were restricted from the year 2000 to ensure that only contemporary data on exposure and morbidity were used to inform the review.

\section{Inclusion and exclusion criteria}

A single assessor reviewed the titles and abstracts of all search results and identified empirical studies specifically addressing: (1) the impact of past or current SHS exposure in the home on the health outcomes of non-smokers; and (2) the impact of home exposure as a child on the risk of future smoking behaviour. Studies were limited to the UK only.

Studies of low quality were excluded; low quality was defined as a score of 3 or less on the Newcastle Ottawa scale or admission by the study authors that it was underpowered for the outcomes of interest. A second, independent assessor reviewed all studies deemed potentially relevant by the first reviewer and confirmed inclusion. Disagreement between the reviewers was resolved through arbitration by a third party.

The study characteristics, population demographic data and outcomes of interest were extracted systematically from each included study by one reviewer and verified by a second, independent reviewer. Due to the differing populations across included studies and variation in the outcomes extracted across the research objectives, it was not possible to combine the results using statistical analysis; a qualitative description of outcomes of interest is given in the results.

\section{Assessment of study quality}

All studies that met the inclusion criteria were critically appraised using the Newcastle-Ottawa scale, as recommended by the Cochrane collaboration for use with nonrandomised, empirical studies [13]. This scale assesses the methodological quality of studies based on three categories: selection of the cohort of interest, comparability of the cohorts, and assessment of the outcomes of interest (for cohort studies) or the assessment of exposure (for casecontrol studies). The optimal assessment of exposure to SHS was considered to be measurement of cotinine levels in blood or saliva, as patient and parent-reported measures of SHS exposure often underestimate the extent of exposure, due to inaccurate reporting of smoking behaviour $[14,15]$.

\section{Results}

The literature searches identified 4151 individual citations once duplicates were removed, and of these 4090 were excluded on title or abstract. After full-text review of the remaining 51 articles, 33 were found to be relevant to the review objectives but 10 of these were excluded for low quality or lack of power; therefore 23 UK studies were included in the review (Figure 1).

The key characteristics for all 23 included studies pertaining to all search objectives are given in Table 1. Further details of the 10 studies excluded for their low quality or lack of statistical power are given in the Additional file 1: Table S1.

\section{The impact of past and current exposure to secondhand smoke in the home}

There were 21 UK studies of sufficient quality that assessed risk of illness or accidents related to past or current exposure to SHS in the home. Of these, 10 were longitudinal cohort, 4 were case control, and 7 were cross-sectional studies. Methodological details of the studies are presented in Table 1. All longitudinal studies scored 6 or above out of a possible 9 on the Newcastle-Ottawa scale. The highest degree of variability for all types of study was the lack of appropriate measure of SHS exposure $[16,28,32,35,36]$. The results are described below in alphabetical order of health condition.

\section{Accidents}

A large longitudinal cohort study of 11,332 children that followed them from birth to age 5 identified a significantly higher risk of accidents in children whose mothers smoked over the 5 year time period, for all accidents and medically attended accidents (Table 2) [16].

\section{Asthma and respiratory symptoms}

Eleven studies were included that reported on the association of SHS exposure (past or current) with asthma and other respiratory symptoms. Two additional studies reported on the association of exposure to SHS with the use of the healthcare system by asthmatic children.

Of the 6 studies identified that reported on nonsmokers who have been exposed to SHS in the home in the past, 5 reported results from the Isle of Wight birth cohort study. The Isle of Wight birth cohort study was a prospective study to indentify risk factors relevant to wheezing and asthma. Of 1536 children born on the Isle of Wight between January 1st 1989 and February 28th 1990, 1456 children were enrolled. Enrolment took place at birth with information on family history of allergies, household pets, smoking habits, birth weight and social class being recorded. The children were followed up at the ages of $1,2,4$ and 10 years [17-21]. In the sub-study of all children included at the 10 year assessment, 


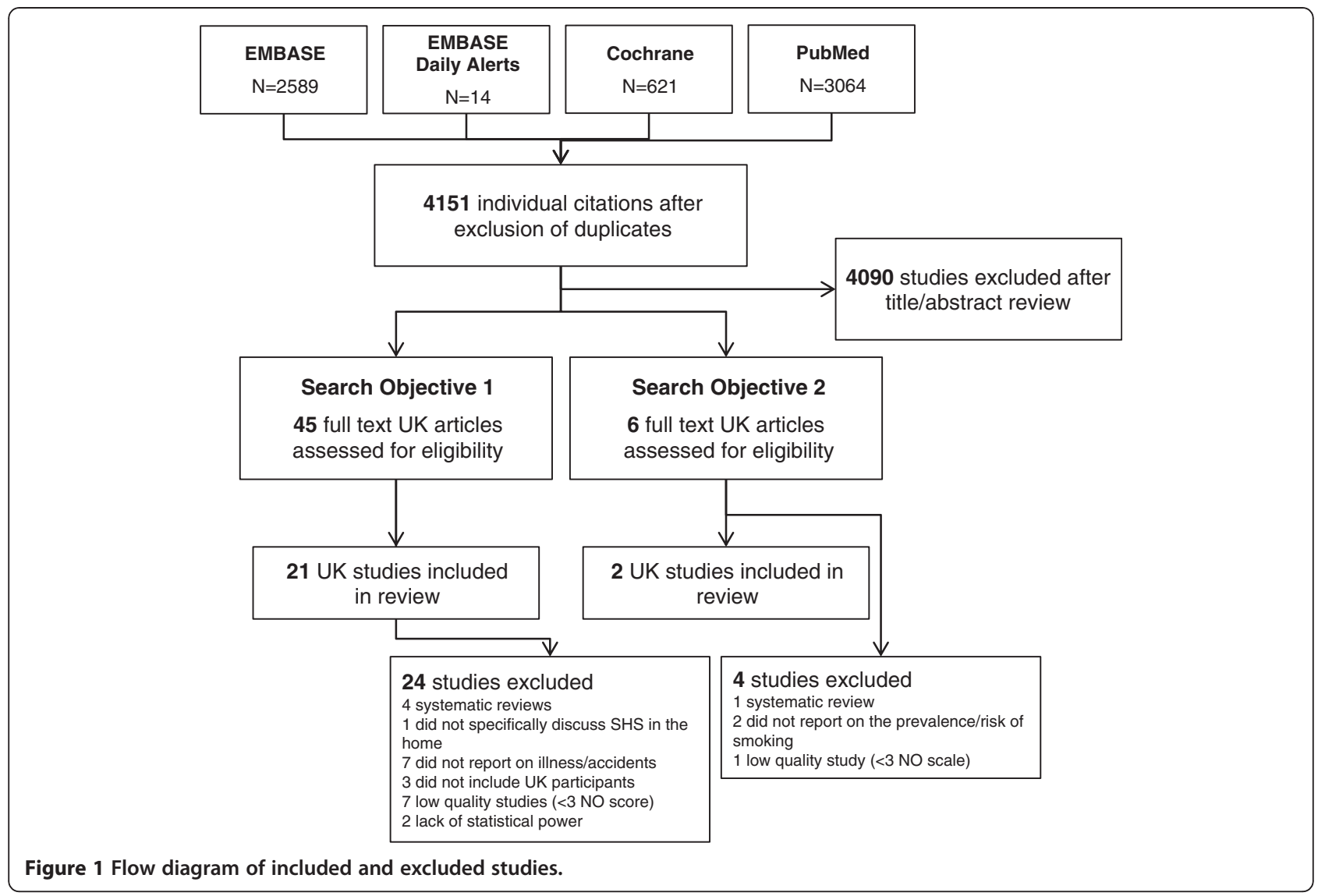

parental smoking at age 1 was significantly associated with currently diagnosed asthma at age $10(\mathrm{OR}=1.99$ [95\% CI 1.15-3.45]; $\mathrm{p}=0.014$ ) and parental smoking at age 4 was found to be significantly associated with current wheeze at age $10(\mathrm{OR}=2.18$ [95\% CI 1.25-3.81]; $\mathrm{p}=0.006$; Table 2) [17]. Parental smoking at birth, 1 year, 2 years and 4 years was significantly associated with early-onset persistent wheeze (Table 2) [19,20]. Symptom expression in bronchial hyper-responsiveness, a hallmark of asthma, at 10 years was also significantly associated with parental smoking at age 4 (Table 2) [18]. However, no significant association was found between exposure to SHS in the home in early childhood (birth to 4 years) and respiratory allergic outcomes at 4 years old (Table 2) [21]. In a separate prospective cohort study, maternal smoking in the home at 30 months was associated with a significantly increased likelihood of any wheeze $(\mathrm{OR}=2.04$ [95\% CI 1.10-3.81]; $\mathrm{p}=0.024)$ and exercise-induced wheeze $(\mathrm{OR}=2.14$ [95\% CI 1.11-4.12]; $\mathrm{p}=0.022)$, but not night cough (OR 1.62 [95\% CI 0.91-2.87]; $\mathrm{p}=0.098$ ), at 6 years (Table 2) [22]. These studies indicate how past smoking behaviour can be associated with future poor respiratory health.

Five studies were identified that reported on the association between current exposure to SHS in the home and asthma or respiratory symptoms. However, it should be noted that causality between current exposure and respiratory symptoms cannot be shown, as prior exposure and other factors may have influenced the health outcomes. In a cohort of high risk children where both parents demonstrated a predisposition toward developing allergic hypersensitivity there were increased odds of reporting wheeze ever and in the first year of life in children whose mother had smoked postnatally (wheeze ever: $\mathrm{OR}=1.93$ [95\% CI 1.10-3.38]; $\mathrm{p}=0.02$, wheeze in first year of life: $\mathrm{OR}=1.79$ [95\% CI 1.05-3.08]; $\mathrm{p}=0.03$; Table 2) [23]. In a large cross-sectional survey of a random sample of adults, those exposed to SHS in the home were significantly more likely to report severe respiratory symptoms compared to those with no exposure [24], whilst two other cross-sectional studies also showed a non-significant decrease in lung function associated with an increase in exposure to SHS in the home (Table 2) $[25,26]$. However, in a case control study that examined the difference in concentrations of indoor pollutants in the homes of children with asthma (cases) and those without (controls), no significant difference in concentrations of any indoor pollutant, including SHS specific particles, was found between asthma case and control environments (Table 2) [27]. 
Table 1 Overview of the main characteristics for UK studies that report evidence on the impact of SHS exposure in the home on health and behavioural outcomes in non-smoker occupants

\begin{tabular}{|c|c|c|c|c|c|c|}
\hline Study ID & $\begin{array}{l}\text { Type of } \\
\text { Study }\end{array}$ & $\begin{array}{l}\text { Included } \\
\text { participants }\end{array}$ & Patient population & $\begin{array}{l}\text { Assessment of SHS } \\
\text { Exposure }\end{array}$ & Disease/Key Outcome & $\begin{array}{l}\mathrm{N}-\mathrm{O} \\
\text { Score }\end{array}$ \\
\hline \multicolumn{7}{|c|}{ Health outcomes of non-smokers previously exposed to SHS in the home (Objective 1) } \\
\hline $\begin{array}{l}\text { Reading et al. } \\
2008[16]\end{array}$ & $\begin{array}{l}\text { Longitudinal } \\
\text { cohort }\end{array}$ & 11,332 families & $\begin{array}{l}\text { Families recruited in the } \\
\text { ASLPAC study }\end{array}$ & $\begin{array}{l}\text { Parent-completed postal } \\
\text { questionnaire }\end{array}$ & Accidents & 6 \\
\hline $\begin{array}{l}\text { Isle of Wight } \\
\text { Birth Cohort }\end{array}$ & $\begin{array}{l}\text { Longitudinal } \\
\text { cohort }\end{array}$ & 1456 & Birth cohort & $\begin{array}{l}\text { Parent-completed } \\
\text { questionnaire }\end{array}$ & $\begin{array}{l}\text { Asthma/wheeze } \\
\text { outcomes }\end{array}$ & 7 \\
\hline $\begin{array}{l}\text { Arshad et al. } \\
2005[17]\end{array}$ & & 1,373 & \multicolumn{2}{|l|}{ Completed 10 year assessment } & & 6 \\
\hline $\begin{array}{l}\text { Kurukulaaratchy } \\
\text { et al. } 2003[18]\end{array}$ & & 169 & \multicolumn{2}{|c|}{ Positive for bronchial hyper-responsiveness at age 10} & & 6 \\
\hline $\begin{array}{l}\text { Kurukulaaratchy } \\
\text { et al. } 2004 \text { [19] }\end{array}$ & & 206 & \multicolumn{2}{|c|}{ Early or late onset persistent wheeze at age 10} & & 6 \\
\hline $\begin{array}{l}\text { Kurukulaaratchy } \\
\text { et al. } 2006[20]\end{array}$ & & 340 & \multicolumn{2}{|c|}{ Reported wheeze ever up to age 10 with atopic parents } & & 6 \\
\hline $\begin{array}{l}\text { Tariq et al. } \\
2000[21]\end{array}$ & & 1,218 & \multicolumn{2}{|l|}{ Completed 4 year assessment } & & 7 \\
\hline $\begin{array}{l}\text { Hennessy et al. } \\
2008[22]\end{array}$ & $\begin{array}{l}\text { Longitudinal } \\
\text { cohort }\end{array}$ & 283 & $\begin{array}{l}\text { Babies born at } 25 \text { weeks } \\
\text { gestation or less }\end{array}$ & $\begin{array}{l}\text { Parent-completed } \\
\text { questionnaire }\end{array}$ & Wheeze & 7 \\
\hline $\begin{array}{l}\text { Murray et al. } \\
2004[23]\end{array}$ & $\begin{array}{l}\text { Longitudinal } \\
\text { cohort }\end{array}$ & 369 & $\begin{array}{l}\text { Child ( }<3 \text { yrs) of parents with } \\
\text { high risk of atopy }\end{array}$ & $\begin{array}{l}\text { Interviewer administered } \\
\text { respiratory questionnaire }\end{array}$ & Wheeze & 6 \\
\hline $\begin{array}{l}\text { Trinder et al. } \\
2000[24]\end{array}$ & $\begin{array}{l}\text { Cross- } \\
\text { sectional } \\
\text { survey }\end{array}$ & 2996 & Adults (16+yrs) & $\begin{array}{l}\text { Patient-completed } \\
\text { questionnaire }\end{array}$ & Respiratory symptoms & 5 \\
\hline $\begin{array}{l}\text { Chen et al. } \\
2001[25]\end{array}$ & $\begin{array}{l}\text { Cross } \\
\text { sectional } \\
\text { survey }\end{array}$ & 301 & $\begin{array}{l}\text { Never smoker adults } \\
(25-64 \text { yrs })\end{array}$ & $\begin{array}{l}\text { Patient-completed } \\
\text { questionnaire }\end{array}$ & Lung Function & 4 \\
\hline $\begin{array}{l}\text { Palmer et al. } \\
2004[26]\end{array}$ & $\begin{array}{l}\text { Cross- } \\
\text { sectional } \\
\text { study }\end{array}$ & 499 & $\begin{array}{l}\text { Child (3-21 yrs) diagnosed } \\
\text { with asthma }\end{array}$ & $\begin{array}{l}\text { Parent-completed } \\
\text { questionnaire }\end{array}$ & Asthma & 4 \\
\hline \multirow{2}{*}{$\begin{array}{l}\text { Gee et al. } \\
2005[27]\end{array}$} & \multirow{2}{*}{$\begin{array}{l}\text { Case-control } \\
\text { study }\end{array}$} & 95 controls & \multirow[t]{2}{*}{ Child (4-16 yrs) } & \multirow{2}{*}{$\begin{array}{l}\text { Air sampling (RSPs, tobacco } \\
\text { specific particles, VOCs, NO2, } \\
\text { formaldehyde) }\end{array}$} & \multirow[t]{2}{*}{ Asthma } & \multirow[t]{2}{*}{5} \\
\hline & & 105 cases & & & & \\
\hline \multirow{2}{*}{$\begin{array}{l}\text { Forbes et al. } \\
2007 \text { [28] }\end{array}$} & \multirow{2}{*}{$\begin{array}{l}\text { Case-control } \\
\text { study }\end{array}$} & 394 controls & \multirow[t]{2}{*}{ Child (3-14 yrs) } & \multirow{2}{*}{$\begin{array}{l}\text { Parent-completed } \\
\text { questionnaire }\end{array}$} & \multirow{2}{*}{$\begin{array}{l}\text { Asthma; Accident \& } \\
\text { Emergency attendance }\end{array}$} & \multirow[t]{2}{*}{5} \\
\hline & & 1018 cases & & & & \\
\hline \multirow{2}{*}{$\begin{array}{l}\text { Crombie et al. } \\
2001[29]\end{array}$} & \multirow{2}{*}{$\begin{array}{l}\text { Cross- } \\
\text { sectional } \\
\text { study }\end{array}$} & 501 families/ & \multirow{2}{*}{$\begin{array}{l}\text { Child ( } 2-12 \text { yrs) diagnosed } \\
\text { with asthma }\end{array}$} & \multirow{2}{*}{$\begin{array}{l}\text { Saliva cotinine assessment; } \\
\text { parent-completed } \\
\text { questionnaire }\end{array}$} & \multirow[t]{2}{*}{ Asthma } & \multirow[t]{2}{*}{4} \\
\hline & & 438 children & & & & \\
\hline $\begin{array}{l}\text { Ward et al. } \\
2007[30]\end{array}$ & $\begin{array}{l}\text { Longitudinal } \\
\text { retrospective } \\
\text { cohort }\end{array}$ & 16,756 parents & Neonates & Parent interview & Birth weight & 7 \\
\hline $\begin{array}{l}\text { Macdonald- } \\
\text { Wallis et al. } \\
2011[31]\end{array}$ & $\begin{array}{l}\text { Longitudinal } \\
\text { cohort }\end{array}$ & 7121 & Birth cohort & $\begin{array}{l}\text { Parent-completed } \\
\text { questionnaire }\end{array}$ & Bone characteristics & 8 \\
\hline \multirow{2}{*}{$\begin{array}{l}\text { Roddam et al. } \\
2007[32]\end{array}$} & \multirow{2}{*}{$\begin{array}{l}\text { Case-control } \\
\text { study }\end{array}$} & 640 controls & \multirow{2}{*}{$\begin{array}{l}\text { Woman (aged } 36-45 \text { years) } \\
\text { diagnosed with breast cancer } \\
\text { from 1987-1990 }\end{array}$} & \multirow[t]{2}{*}{ Patient interview } & \multirow[t]{2}{*}{ Breast cancer } & \multirow[t]{2}{*}{4} \\
\hline & & 639 cases & & & & \\
\hline $\begin{array}{l}\text { Llewellyn et al. } \\
2009[33]\end{array}$ & $\begin{array}{l}\text { Cross- } \\
\text { sectional } \\
\text { survey }\end{array}$ & 4809 & $\begin{array}{l}\text { Non-smoking adults } \\
\text { ( }>50 \text { years) }\end{array}$ & Saliva cotinine & Cognitive impairment & 5 \\
\hline $\begin{array}{l}\text { Williams et al. } \\
2000[34]\end{array}$ & $\begin{array}{l}\text { Cross- } \\
\text { sectional } \\
\text { survey }\end{array}$ & 763 & Child (1.5-4.5 years) & $\begin{array}{l}\text { Parent-completed } \\
\text { questionnaire }\end{array}$ & Dental caries & 4 \\
\hline $\begin{array}{l}\text { MacLennan et al. } \\
2006 \text { [35] }\end{array}$ & $\begin{array}{l}\text { Longitudinal } \\
\text { cohort }\end{array}$ & 13919 & Adolescent (15-19 years) & Patient interview & Meningococcal carriage & 6 \\
\hline
\end{tabular}


Table 1 Overview of the main characteristics for UK studies that report evidence on the impact of SHS exposure in the home on health and behavioural outcomes in non-smoker occupants (Continued)

\begin{tabular}{|c|c|c|c|c|c|c|}
\hline $\begin{array}{l}\text { Coen et al. } \\
2006 \text { [36] }\end{array}$ & $\begin{array}{l}\text { Case-control } \\
\text { study }\end{array}$ & $\begin{array}{l}144 \text { survivors } \\
144 \text { matched } \\
\text { controls }\end{array}$ & Adolescent (15-19 yrs) & Patient interview & Meningococcal disease & 4 \\
\hline \multicolumn{7}{|c|}{ Risk of future smoking in children exposed to second hand smoke in the home (Objective 2) } \\
\hline \multirow{4}{*}{$\begin{array}{l}\text { Milton et al. } \\
2004[37]\end{array}$} & \multirow{4}{*}{$\begin{array}{l}\text { Longitudinal } \\
\text { cohort }\end{array}$} & 247 at aged 9 & \multirow{4}{*}{$\begin{array}{l}\text { Primary school children aged } \\
9-11\end{array}$} & \multirow{4}{*}{$\begin{array}{l}\text { Both child and parent (usually } \\
\text { mother) postal questionnaires }\end{array}$} & \multirow{4}{*}{$\begin{array}{l}\text { Smoking behaviour } \\
\text { assessed by self- } \\
\text { completed questionnaire }\end{array}$} & \multirow[t]{4}{*}{9} \\
\hline & & 257 at aged & & & & \\
\hline & & 10 & & & & \\
\hline & & 239 at aged 11 & & & & \\
\hline $\begin{array}{l}\text { Griesbach et al. } \\
2003 \text { [38] }\end{array}$ & $\begin{array}{l}\text { Cross- } \\
\text { sectional } \\
\text { survey }\end{array}$ & 3132 & $\begin{array}{l}\text { Adolescents aged } 15+\text { from } \\
\text { Scotland }(N=1724) \text { and Wales } \\
(N=1408)\end{array}$ & $\begin{array}{l}\text { Self-completed questionnaire } \\
\text { on parents and other smokers } \\
\text { in the home }\end{array}$ & $\begin{array}{l}\text { Smoking behaviour } \\
\text { assessed by self- } \\
\text { completed questionnaire }\end{array}$ & 4 \\
\hline
\end{tabular}

$\mathrm{N}-\mathrm{O}$ Score, Newcastle-Ottawa score(Wells et al.). A higher score indicates higher methodological quality; max. 9.

$\mathrm{RSP}$, respirable suspended particle; VOC, volatile organic compound.

In a case control study, no association was found between asthma patients' Accident and Emergency (A\&E) attendance and exposure to SHS in UK homes [28]. However, another cross-sectional study found that in asthmatic children, a reduction in the rate of health service contacts was significantly associated with increased number of cigarettes smoked in the home (Table 2) [29]. The explanation given by the study authors for the observed fewer health service contacts was that heavy smoking may reduce the awareness of parents to the child's asthmatic symptoms.

\section{Birth outcomes}

One large retrospective birth cohort study demonstrated that for SHS exposed non-smoking mothers, there was a significant decrease in the crude and adjusted mean birth weight of their offspring compared to non SHS exposed mothers (crude mean difference: $-0.059 \mathrm{~kg}, \mathrm{p}<0.001$; adjusted mean difference: $-0.036 \mathrm{~kg}, \mathrm{p}=0.025$ ) [30]. Exposure to SHS was also associated with non-significant increases in the incidence of premature births (Table 2).

\section{Bone characteristics}

A longitudinal birth cohort study found that paternal smoking during pregnancy at weeks 18 and 32 was associated with a significant increase in total body-less-head bone area in girls but not boys at age 10 (Table 2) [31]. The main conclusion made by the authors was that the significant influence that paternal smoking factors had on girls' health outcomes, combined with non-significant maternal smoking outcomes, could indicate that these associations are largely driven by familial characteristics related to childhood, and are unlikely to be due to intrauterine mechanisms.

\section{Breast cancer}

A UK case-control study of women aged 36-45 years who were diagnosed with invasive breast cancer reported no increased risk of breast cancer associated with exposure to SHS in the home through partner smoking (Table 2) [32].

\section{Cognitive impairment}

A large, cross-sectional study conducted in a cohort of non-smoking adults over 50 years of age identified a statistically significant increased odds of cognitive impairment associated with rising saliva cotinine levels in nonsmokers ( $\mathrm{p}=0.02$ for trend; OR = 1.44; Table 2) [33].

\section{Dental caries}

A cross-sectional survey demonstrated a significant increase in the odds of caries in pre-school children who had a smoking mother and were from families who had manual occupations (Table 2) [34].

\section{Meningitis carriage and disease}

Two studies, one case control and one longitudinal, were identified that reported an association between SHS exposure in the home and a significantly increased likelihood of meningococcal carriage and disease in adolescents, respectively $[35,36]$. Exposure to other smokers in the home was a significant factor for a positive test for meningococcal carriage according to a multivariable analysis (Table 2) [35]. Exposure to smokers was also found to be significantly and independently associated with meningococcal disease (Table 2), but this association is likely to be due to higher carriage rates in smokers rather than SHS exposure [36].

\section{The impact of secondhand smoke exposure on smoking} behaviour

\section{Summary of relevant studies}

Only 2 UK studies were identified for inclusion that discussed the impact of exposure as a child on the risk of current or future smoking behaviour [37,38]. Of these studies, 1 was longitudinal, which scored the maximum 
Table 2 Health outcomes of non-smokers exposed to SHS in the home

\begin{tabular}{|c|c|c|c|c|c|}
\hline Study ID & $\mathbf{N}$ & \multicolumn{2}{|l|}{ Outcome } & $\begin{array}{l}\text { OR/RR for SHS exposure } \\
\text { compared to non- } \\
\text { exposure }\end{array}$ & $P$ value \\
\hline \multicolumn{6}{|l|}{ Accidents } \\
\hline \multirow{3}{*}{$\begin{array}{l}\text { Reading et al. } \\
2008[16]\end{array}$} & \multirow{3}{*}{$\begin{array}{l}11,332 \\
\text { families }\end{array}$} & \multicolumn{4}{|l|}{ For maternal smoking vs. non-smoking: } \\
\hline & & \multicolumn{2}{|l|}{ All accidents } & RR $1.17,95 \%$ Cl 1.12-1.23 & $<0.01$ \\
\hline & & \multicolumn{2}{|l|}{ Medically attended accidents } & RR $1.23,95 \%$ Cl $1.14-1.32$ & $<0.01$ \\
\hline \multicolumn{6}{|c|}{ Asthma and Related Symptoms } \\
\hline \multicolumn{6}{|c|}{ Isle of Wight Birth Cohort } \\
\hline \multirow{2}{*}{$\begin{array}{l}\text { Arshad et al. } \\
2005[17]\end{array}$} & \multirow[t]{2}{*}{1,373} & \multicolumn{2}{|c|}{ Asthma at age 10 by parental smoking at age 1 vs. non-smoking } & OR 1.99; 95\% Cl 1.15-3.45, & 0.014 \\
\hline & & \multicolumn{2}{|c|}{ Wheeze at age 10 by parental smoking at age 4 vs. non-smoking } & OR $2.18 ; 95 \% \mathrm{Cl} 1.25-3.81$ & 0.006 \\
\hline $\begin{array}{l}\text { Kurukulaaratchy } \\
\text { et al. } 2003[18]\end{array}$ & 169 & \multicolumn{2}{|c|}{$\begin{array}{l}\text { Bronchial hyper-responsiveness at } 10 \text { years by parental smoking at } \\
\text { age } 4 \text { vs. non-smoking }\end{array}$} & OR $2.62,95 \% \mathrm{Cl} 1.03-6.71$ & 0.04 \\
\hline \multirow[t]{4}{*}{$\begin{array}{l}\text { Kurukulaaratchy } \\
\text { et al. } 2004[19]\end{array}$} & 206 & $\begin{array}{l}\text { Early-onset persistent wheeze at age } 10 \text { vs. no } \\
\text { parental smoking: }\end{array}$ & $\begin{array}{l}\text { by parental } \\
\text { smoking at } \\
\text { birth }\end{array}$ & OR $1.88,95 \% \mathrm{Cl} 1.27-2.77$ & 0.001 \\
\hline & & & $\begin{array}{l}\text { by parental } \\
\text { smoking at } \\
1 \text { year }\end{array}$ & OR $2.05,95 \%$ Cl 1.39-3.02 & $<0.001$ \\
\hline & & & $\begin{array}{l}\text { by parental } \\
\text { smoking at } \\
2 \text { years }\end{array}$ & OR $2.00,95 \% \mathrm{Cl} 1.33-3.00$ & 0.001 \\
\hline & & & $\begin{array}{l}\text { by parental } \\
\text { smoking at } \\
4 \text { years }\end{array}$ & OR $2.25,95 \%$ Cl 1.52-3.32 & $<0.001$ \\
\hline \multirow[t]{3}{*}{$\begin{array}{l}\text { Kurukulaaratchy } \\
\text { et al. } 2006[20]\end{array}$} & 340 & Wheeze ever vs. no parental smoking & $\begin{array}{l}\text { by parental } \\
\text { smoking at } \\
1 \text { years }\end{array}$ & OR $1.54,95 \%$ Cl 1.07-2.22 & 0.02 \\
\hline & & & $\begin{array}{l}\text { by parental } \\
\text { smoking at } \\
2 \text { years }\end{array}$ & OR $1.51,95 \%$ Cl 1.04-2.21 & 0.03 \\
\hline & & & $\begin{array}{l}\text { by parental } \\
\text { smoking at } \\
4 \text { years }\end{array}$ & OR $1.80,95 \%$ Cl 1.25-2.58 & 0.001 \\
\hline
\end{tabular}

\begin{tabular}{|c|c|c|c|c|}
\hline \multirow[t]{2}{*}{$\begin{array}{l}\text { Tariq et al. } \\
2000[21]\end{array}$} & \multirow[t]{2}{*}{1,218} & $\begin{array}{l}\text { Asthma at } 4 \text { years by exposure to SHS in the home in early childhood } \\
\text { vs. no exposure }\end{array}$ & OR $1.2,95 \%$ Cl 0.3-2.7 & NR \\
\hline & & $\begin{array}{l}\text { Any allergic hypersensitivity at } 4 \text { years by exposure to SHS in the } \\
\text { home in early childhood vs. no exposure }\end{array}$ & OR $1.1,95 \%$ Cl 0.6-1.6 & NR \\
\hline \multicolumn{5}{|l|}{ Other studies } \\
\hline \multirow{3}{*}{$\begin{array}{l}\text { Hennessy et al. } \\
2008[22]\end{array}$} & 219 & Any wheeze at 6 years by exposure at 30 months vs. no exposure & OR $2.04,95 \% \mathrm{Cl} 1.10-3.81$ & 0.024 \\
\hline & 218 & $\begin{array}{l}\text { Exercise-induced wheeze at } 6 \text { years by exposure at } 30 \text { months vs. no } \\
\text { exposure }\end{array}$ & OR $2.14,95 \% \mathrm{Cl} 1.11-4.12$ & 0.022 \\
\hline & 219 & Night cough at 6 years by exposure at 30 months vs. no exposure) & OR $1.62,95 \% \mathrm{Cl} 0.91-2.87$ & 0.098 \\
\hline \multirow{3}{*}{$\begin{array}{l}\text { Murray et al. } \\
2004[23]\end{array}$} & 369 & \multicolumn{3}{|l|}{ For mother smoking postnatally vs. non-smoking } \\
\hline & & Wheeze ever & $\begin{array}{l}\text { OR } 1.93,95 \% \text { Cl } 1.10-3.38 \\
\text { (adjusted analysis) }\end{array}$ & 0.02 \\
\hline & & $\begin{array}{l}\text { Wheeze in first } \\
\text { year }\end{array}$ & $\begin{array}{l}\text { OR } 1.79,95 \% \text { Cl } 1.05-3.08 \\
\text { (adjusted analysis) }\end{array}$ & 0.03 \\
\hline $\begin{array}{l}\text { Trinder et al. } \\
2000[24]\end{array}$ & 2996 & Severe respiratory symptoms & OR $1.4,95 \% \mathrm{Cl} 1.0-1.8$ & NR \\
\hline $\begin{array}{l}\text { Chen et al. } \\
2001[25]\end{array}$ & 301 & Lung function: mean residuals of $\mathrm{FEV}_{1}$ and FVC & NR & $>0.05$ \\
\hline $\begin{array}{l}\text { Palmer et al. } \\
2006[26]\end{array}$ & 504 & Lung function & NR & $>0.05$ \\
\hline
\end{tabular}


Table 2 Health outcomes of non-smokers exposed to SHS in the home (Continued)

\begin{tabular}{|c|c|c|c|c|c|}
\hline \multirow{2}{*}{$\begin{array}{l}\text { Gee et al. } \\
2005[27]\end{array}$} & & \multirow{2}{*}{\multicolumn{2}{|c|}{$\begin{array}{l}\text { Difference in indoor pollutant levels between asthma cases and } \\
\text { controls }\end{array}$}} & \multirow[t]{2}{*}{ NR } & \multirow{2}{*}{$\begin{array}{l}>0.05 \text { for all } \\
\text { pollutants }\end{array}$} \\
\hline & 105 cases & & & & \\
\hline \multirow[t]{2}{*}{$\begin{array}{l}\text { Forbes et al. } \\
2007[28]\end{array}$} & $\begin{array}{l}394 \\
\text { controls }\end{array}$ & \multirow{2}{*}{\multicolumn{2}{|c|}{ A\&E attendance in asthma patients }} & \multirow[t]{2}{*}{$\begin{array}{l}\text { OR } 1.12,95 \% \mathrm{Cl} 0.80-1.58 \\
\text { (adjusted analysis) }\end{array}$} & \multirow[t]{2}{*}{ NR } \\
\hline & 1018 cases & & & & \\
\hline \multirow[t]{4}{*}{$\begin{array}{l}\text { Crombie et al. } \\
2001[29]\end{array}$} & $\begin{array}{l}501 \\
\text { families }\end{array}$ & \multirow{4}{*}{$\begin{array}{l}\text { Health service contacts for asthma by number of } \\
\text { cigarettes smoked by parent per day (compared to } \\
0-5 \text { ) }\end{array}$} & $6-10$ & IRR $0.81,95 \%$ Cl 0.71-0.92 & \multirow[t]{4}{*}{$\begin{array}{l}0.0002 \text { for } \\
\text { trend }\end{array}$} \\
\hline & \multirow{3}{*}{$\begin{array}{l}438 \\
\text { children }\end{array}$} & & $11-15$ & IRR $0.70,95 \%$ Cl 0.59-0.83 & \\
\hline & & & $16-20$ & IRR $0.74,95 \%$ Cl $0.61-0.91$ & \\
\hline & & & $>20$ & IRR $0.66,95 \%$ Cl 0.47-0.93 & \\
\hline \multicolumn{6}{|l|}{ Birth weight } \\
\hline \multirow{2}{*}{$\begin{array}{l}\text { Ward et al. } \\
2007[30]\end{array}$} & \multirow{2}{*}{$\begin{array}{l}16,756 \\
\text { parents }\end{array}$} & \multirow{2}{*}{$\begin{array}{l}\text { Mean birth weight }(\mathrm{kg}) \text { difference between SHS } \\
\text { exposed and non-exposed non smoking mothers }\end{array}$} & crude & \multirow[t]{2}{*}{ NR } & $<0.001$ \\
\hline & & & adjusted & & 0.025 \\
\hline \multicolumn{6}{|l|}{ Bone Characteristics } \\
\hline $\begin{array}{l}\text { Macdonald- } \\
\text { Wallis et al. } \\
2011[31]\end{array}$ & 3591 & \multicolumn{2}{|c|}{$\begin{array}{l}\text { TBLH bone area in girls at age } 10 \text { by paternal smoking during } \\
\text { pregnancy vs. no smoking }\end{array}$} & NA & $\begin{array}{l}0.029 \text { (fully } \\
\text { adjusted } \\
\text { analysis) }\end{array}$ \\
\hline \multicolumn{6}{|l|}{ Breast Cancer } \\
\hline $\begin{array}{l}\text { Roddam et al. } \\
2007[32]\end{array}$ & $\begin{array}{l}640 \\
\text { controls, } \\
639 \text { cases }\end{array}$ & \multicolumn{2}{|l|}{ Breast cancer in never smokers } & RR $0.89,95 \%$ Cl 0.64-1.25 & NR \\
\hline \multicolumn{6}{|l|}{ Cognitive Impairment } \\
\hline \multirow[t]{4}{*}{$\begin{array}{l}\text { Llewellyn et al. } \\
2009[33]\end{array}$} & 4809 & \multicolumn{2}{|c|}{$\begin{array}{l}\text { Cognitive impairment by cotinine level quartile in non-smoker } \\
\text { (compared to lowest quartile): }\end{array}$} & & \multirow{4}{*}{0.02 for trenc } \\
\hline & & & $\begin{array}{l}\text { Second } \\
\text { quartile } \\
\text { cotinine level }\end{array}$ & OR $1.08,95 \%$ Cl 0.78-1.48 & \\
\hline & & & $\begin{array}{l}\text { Third quartile } \\
\text { cotinine level }\end{array}$ & OR $1.13,95 \%$ Cl 0.81-1.56 & \\
\hline & & & $\begin{array}{l}\text { Fourth quartile } \\
\text { cotinine level }\end{array}$ & OR $1.44,95 \%$ Cl 1.07-1.94 & \\
\hline \multicolumn{6}{|l|}{ Dental Caries } \\
\hline \multirow{3}{*}{$\begin{array}{l}\text { Williams et al. } \\
2000[34]\end{array}$} & 763 & \multirow[t]{3}{*}{ Dental caries } & & & \\
\hline & & & $\begin{array}{l}\text { Non-manual } \\
\text { occupations } \\
(n=458)\end{array}$ & OR $1.96,1.00-3.85$ & 0.05 \\
\hline & & & $\begin{array}{l}\text { Manual } \\
\text { occupations } \\
(\mathrm{n}=280)\end{array}$ & OR $1.55,1.02-2.35$ & $<0.05$ \\
\hline \multicolumn{6}{|c|}{ Meningitis Carriage and Disease } \\
\hline \multirow{2}{*}{$\begin{array}{l}\text { MacLennan et al. } \\
2006[35]\end{array}$} & 13,919 & \multirow{2}{*}{\multicolumn{2}{|c|}{$\begin{array}{l}\text { Meningococcal carriage in exposed }(n=5064) \\
\text { vs. non-exposed }(n=8547)\end{array}$}} & \multirow[t]{2}{*}{ OR $1.17,95 \%$ Cl 1.05-1.30 } & 0.004 \\
\hline & & & & & \\
\hline Coen 2006 [36] & $\begin{array}{l}144 \\
\text { survivors }\end{array}$ & Meningococcal disease in adolescents exposed to si & okers & OR $1.83 ; 95 \%$ Cl 1.0-3.3 & 0.01 \\
\hline & $\begin{array}{l}144 \\
\text { matched } \\
\text { controls }\end{array}$ & & & & \\
\hline
\end{tabular}

A\&E, accident and emergency; $\mathrm{Cl}$, confidence interval; IFEV, forced expiratory volume; FVC, forced vital capacity; RR, incidence rate ratio; NA, not appropriate; NR, not reported; $\mathrm{OR}$, odds ratio; $\mathrm{RR}$, risk ratio; $\mathrm{TBLH}$, total body less head. 
score of 9 on the Newcastle-Ottawa scale, the other was a cross-sectional cohort study scoring 4 . The key outcomes assessed were different frequencies of smoking: ever having tried smoking or daily smoking $[37,38]$.

\section{Impact on smoking behaviour}

A longitudinal study found that significantly more children aged 10 and 11 had tried smoking if they lived with a smoker $(\mathrm{p}<0.001$; Table 3$)$ compared to those who lived in a non-smoking household [37]. There was also a significant association between having tried smoking and living with a mother or sibling who smokes (Table 3) [37]. Exposure to SHS was not, however, found to be significantly associated with smoking behaviour [37]. A cross-sectional survey in Scotland and Wales also found that the presence of a parent smoker or other smoker was associated with a significantly increased likelihood of being a daily smoker compared with neither parent smoking or living with no smokers $(\mathrm{p}<0.001$ for all analyses; Table 3) [38].

Regarding future smoking, a logistic regression analysis from the longitudinal study found that having a father or brother who smoked when aged 9 meant that the child was over 5 times more likely to try smoking by age 11 than those who did not have a smoking father or brother at age 9 (Table 3) [37].

\section{Discussion-conclusions}

Empirical UK evidence identified in this review has demonstrated that there are significant negative health outcomes associated with SHS exposure in the home, such as asthma, meningococcal disease, cognitive impairment, and dental caries.

In addition to the potential negative impact on health outcomes, SHS exposure in the home also seems to be related to smoking behaviour, particularly in children and adolescents. A significant proportion of children and adolescents in the UK who live in smoking households have tried smoking themselves, and living in a smoking household has been shown to be associated with an increased likelihood of regular smoking in the future [37,38]. Interestingly, time spent in a 'smoky environment' was not associated with ever trying smoking, although the same study showed that living with a smoker significantly increased the probability of ever trying smoking [37]. This suggests that the influence of smoking in the home is not only related to SHS exposure in itself; even if smoking members of the house are careful to smoke out of the direct environment

Table 3 Risk of future smoking in children exposed to second hand smoke in the home

\begin{tabular}{|c|c|c|c|c|}
\hline Study ID & Behaviour outcome & $\mathbf{N}$ & $\begin{array}{l}\text { OR for exposure compared to } \\
\text { non-exposure }\end{array}$ & $P$ value \\
\hline \multirow{10}{*}{$\begin{array}{l}\text { Milton et al. } 2004 \\
\text { [37] }\end{array}$} & Ever tried smoking: & & & \\
\hline & Currently exposed to smokers in the home vs. & $\begin{array}{l}254 \text { at } \\
\text { aged } 10\end{array}$ & NR & $<0.001$ \\
\hline & non-smokers & $\begin{array}{l}238 \text { at } \\
\text { aged } 11\end{array}$ & & $<0.001$ \\
\hline & Currently exposed to SHS in the home vs. not & $\begin{array}{l}256 \text { at } \\
\text { aged } 10\end{array}$ & NR & $\begin{array}{l}0.136 \text { (aged } \\
10)\end{array}$ \\
\hline & exposed & $\begin{array}{l}236 \text { at } \\
\text { aged } 11\end{array}$ & & $\begin{array}{l}0.064 \text { (aged } \\
11)\end{array}$ \\
\hline & Tried smoking by age 11 : & & & \\
\hline & Exposed to smoking father at age 9 & NR & OR $5.27,95 \% \mathrm{Cl} 2.18-12.74$ & 0.002 \\
\hline & vs. non-smoking father & & & \\
\hline & Exposed to smoking brother at age 9 & NR & OR $5.32,95 \% \mathrm{Cl} 1.36-21.18$ & 0.017 \\
\hline & vs. non-smoking brother & & & \\
\hline \multirow[t]{6}{*}{$\begin{array}{l}\text { Griesbach et al. } \\
2003[38]\end{array}$} & $\begin{array}{l}\text { Being a daily smoker by the presence of either one or both } \\
\text { parents who smoke }\end{array}$ & & & \\
\hline & Scotland & 1635 & OR $1.73,95 \% \mathrm{Cl} 1.32-2.26$ & $<0.001$ \\
\hline & Wales & 1364 & OR $1.98,95 \% \mathrm{Cl} 1.46-2.70$ & $<0.001$ \\
\hline & $\begin{array}{l}\text { Being a daily smoker by the presence of other (non-parent) } \\
\text { smoker at home }\end{array}$ & & & \\
\hline & Scotland & 1635 & OR $2.43,95 \% \mathrm{Cl} 1.84-3.22$ & $<0.001$ \\
\hline & Wales & 1364 & OR $2.11,95 \% \mathrm{Cl} 1.47-3.02$ & $<0.001$ \\
\hline
\end{tabular}

$\mathrm{Cl}$, confidence interval; $\mathrm{NR}$, not reported; OR, odds ratio. 
of the non-smoker, there may still be a social or familial influence that encourages children to take up smoking.

The strengths of this review include the comprehensive search strategy, systematic data extraction and robust quality assessment method employed. The limitation of the review to UK studies reduced heterogeneity between study populations, but may have meant that other potential risks associated with SHS exposure in the home that have been identified outside of the UK will have been missed. The generalisability of the review results to countries outside of the UK is also unclear, although it is likely that the results will be relevant to countries that have had a smoking ban in public places for a similar length of time as the UK. There are several other limitations to this review, particularly due to the varied design of the studies that were included; the studies were often not comparable in terms of measurement of exposure to SHS or definition of health outcomes. The quality of each study was individually assessed using the Newcastle-Ottawa scale, and conclusions of the review have been limited to statements based on a good quality evidence base. Due to the difficulty of proving a causal link between SHS and health outcomes, most of the studies that were included reported statistical associations.

This review was limited to second hand smoke exposure only, but in addition to second hand exposure to smoke in the air there is also a risk of 'third hand' exposure, which refers to the residual tobacco smoke particles that remain after a cigarette is extinguished [39]. Children are highly susceptible to the ingestion of these third hand smoke particles [40]. Research conducted in the USA has shown that in smoking households high levels of surface nicotine can persist for several months even when smoking has ceased $[41,42]$.

In summary, a review of the UK data on the impact of exposure to SHS in the home on health outcomes of non-smoker occupants has demonstrated a significantly increased probability of many negative health outcomes. Furthermore, smoking in the home appears to influence the future smoking behaviours of non-smokers. It is unclear how knowledgeable smokers are on the effects of smoking in the home, but the implication of this review is that it is essential that people who currently smoke in their home, particularly those with children or with a pregnant partner, are made aware of the potential impact of their smoking behaviour on non-smokers. Current smokers should be given adequate support to assist them in stopping smoking and reducing exposure of non-smokers to SHS within their home, which could include both counselling and access to pharmacotherapy. The evidence from this review lends support to the rationale and objectives of campaigns such as The Smoke Outside campaign from Smoke Free South West [43] and the Take 7 Steps Out campaign in the North East of the UK [44], both of which aim to educate people on the potential dangers of smoking in the home and to provide encouragement for people to smoke outside.

\section{Additional file}

Additional file 1: Table S1. Overview of the main characteristics for additional studies that report UK evidence on the impact of SHS exposure in the home on health and behavioural outcomes in non-smoker occupants not discussed in the main body of the manuscript.

\section{Abbreviations}

A\&E: Accident and emergency; Cl: Confidence interval; FEV: Forced expiratory volume; FVC: Forced vital capacity; IRR: Incidence risk ratio; NICE: National institute for health and clinical excellence; N-O: Newcastle-Ottawa scale; OR: odds ratio; RR: Risk ratio; RSP: Respirable suspended particle; SHS: Secondhand smoke; TBLH: Total body less head; VOC: Volatile organic compound.

\section{Competing interests}

lan Lockhart is an employee of Pfizer Ltd. Jeanette Kusel is an employee of Costello Medical Consulting and Beth Timm was a former employee, who received funding from Pfizer $L$ td. to conduct the review.

\section{Authors' contributions}

JK and BT conducted the systematic literature review and contributed to the writing of the manuscript. IL reviewed the systematic literature review methods and results and contributed to the writing of the manuscript. All authors read and approved the final manuscript.

\section{Acknowledgements}

This study was funded by Pfizer Ltd.

\section{Author details}

${ }^{1}$ Costello Medical Consulting Ltd, St John's Innovation Centre, Cowley Road, Cambridge CB4 OWS, UK. ${ }^{2}$ Pfizer Ltd, Walton Oaks, Dorking Road, Walton-on-the-Hill, Tadworth KT20 7NS,Surrey, UK.

Received: 17 October 2012 Accepted: 16 January 2013 Published: 29 January 2013

\section{References}

1. World Lung Foundation,American Cancer Society: The Tobacco Atlas, Chapter: Secondhand Smoking. 2012. [cited 2012 August]; Available from: http://www.tobaccoatlas.org/harm/secondhand_smoking/youth/.

2. Holliday JC, Moore GF, Moore LA: Changes in child exposure to secondhand smoke after implementation of smoke-free legislation in Wales: a repeated cross-sectional study. BMC Publ Health 2009, 9:430.

3. Huttunen R, Heikkinen T, Syrjanen J: Smoking and the outcome of infection. J Intern Med 2011, 269:258-269.

4. Jones LL, Hashim A, McKeever T, Cook DG, Britton J, Leonardi-Bee J: Parental and household smoking and the increased risk of bronchitis, bronchiolitis and other lower respiratory infections in infancy: systematic review and meta-analysis. Respir Res 2011, 12:5.

5. Rushton L: Health impact of environmental tobacco smoke in the home. Rev Environ Health 2004, 19:291-309.

6. Stayner L, Bena J, Sasco AJ, Smith R, Steenland K, Kreuzer M, Straif K: Lung cancer risk and workplace exposure to environmental tobacco smoke. Am J Public Health 2007, 97:545-551.

7. Office of National Statistics: General lifestyle survey overview; a report on the 2010 General Lifestyle Survey. 2010, Available from: http://www.ons. gov.uk/ons/rel/ghs/general-lifestyle-survey/2010/index.html.

8. Callinan JE, Clarke A, Doherty K, Kelleher C: Legislative smoking bans for reducing secondhand smoke exposure, smoking prevalence and tobacco consumption. Cochrane Database Syst Rev 2010, 14:CD005992.

9. Healthier Scotland: Clearing The Air. 2006. [cited 2012 June]; Available from: http://www.clearingtheairscotland.com/background/index.html. 
10. Smokefree England. 2007. [cited 2012 June]; Available from: http:// collections.europarchive.org/tna/20110202220654/http://www. smokefreeengland.co.uk.

11. Haw SJ, Gruer L: Changes in exposure of adult non-smokers to secondhand smoke after implementation of smoke-free legislation in Scotland: national cross sectional survey. BMJ 2007, 335:549.

12. Passive Smoking and Children: A Report of the Tobacco Advisory Group of the Royal College of Physicians. 2010. [cited 2012 April]; Available from: http:// bookshop.rcplondon.ac.uk/contents/pub305-e37e88a5-4643-4402-92986936de103266.pdf

13. Wells G, Shea B, O'Connell D, Peterson J, Welch V, Losos M, Tugwell P: The Newcastle-Ottawa Scale (NOS) for assessing the quality of nonrandomised studies in meta-analyses. 2011. [cited 2012 April]; Available from: http:// www.ohri.ca/programs/clinical_epidemiology/oxford.asp.

14. de Lorenze G, Kharrazi M, Kaufman F, Eskenazi B, Bernert JT: Exposure to environmental tobacco smoke in pregnant women: The association between self-report and serum cotinine. Environ Res 2002, 90:21-32.

15. de Chazeron I, Llorca P, Ughetto S, Coudore F, Boussiron D, Perriot J, Vendittelli $F$, Sapin V, Lemery D: Occult maternal exposure to environmental tobacco smoke exposure. Tob Control 2007, 16:64-65

16. Reading R, Jones A, Haynes R, Daras K, Emond A: Individual factors explain neighbourhood variations in accidents to children under 5 years of age. Soc Sci Med 2008, 67:915-927.

17. Arshad SH, Kurukulaaratchy RJ, Fenn M, Matthews S: Early life risk factors for current wheeze, asthma, and bronchial hyperresponsiveness at 10 years of age. Chest 2005, 127:502-508.

18. Kurukulaaratchy RJ, Matthews S, Waterhouse L, Arshad SH: Factors influencing symptom expression in children with bronchial hyperresponsiveness at 10 years of age. J Allergy Clin Immunol 2003, 112:311-316.

19. Kurukulaaratchy RJ, Matthews S, Arshad SH: Does environment mediate earlier onset of the persistent childhood asthma phenotype? Pediatrics 2004, 113:345-350.

20. Kurukulaaratchy RJ, Matthews S, Arshad SH: Relationship between childhood atopy and wheeze: what mediates wheezing in atopic phenotypes? Ann Allergy Asthma Immunol 2006, 97:84-91.

21. Tariq SM, Hakim EA, Matthews SM, Arshad SH: Influence of smoking on asthmatic symptoms and allergen sensitisation in early childhood. Postgrad Med J 2000, 76:694-699.

22. Hennessy EM, Bracewell MA, Wood N, Wolke D, Costeloe K, Gibson A, Marlow N: Respiratory health in pre-school and school age children following extremely preterm birth. Arch Dis Child 2008, 93:1037-1043.

23. Murray CS, Woodcock A, Smillie FI, Cain G, Kissen P, Custovic A: Tobacco smoke exposure, wheeze, and atopy. Pediatr Pulmonol 2004, 37:492-498.

24. Trinder PM, Croft PR, Lewis M: Social class, smoking and the severity of respiratory symptoms in the general population. J Epidemiol Community Health 2000, 54:340-343.

25. Chen R, Tunstall-Pedoe $H$, Tavendale R: Environmental tobacco smoke and lung function in employees who never smoked: the scottish MONICA study. Occup Environ Med 2001, 58:563-568.

26. Palmer CN, Doney AS, Lee SP, Murrie I, Ismail T, Macgregor DF, Mukhopadhyay S: Glutathione S-transferase M1 and P1 genotype, passive smoking, and peak expiratory flow in asthma. Pediatrics 2006, 118:710-716.

27. Gee IL, Watson AFR, Tavernier G, Stewart L, Fletcher G, Niven RML: Indoor air quality, environmental tobacco smoke and asthma: a case control study of asthma in a community population. Indoor and Built Environment 2005, $14: 215-219$.

28. Forbes L, Harvey S, Newson R, Jarvis D, Luczynska C, Price J, Burney P: Risk factors for accident and emergency (A\&E) attendance for asthma in inner city children. Thorax 2007, 62:855-860.

29. Crombie IK, Wright A, Irvine L, Clark RA, Slane PW: Does passive smoking increase the frequency of health service contacts in children with asthma? Thorax 2001, 56:9-12.

30. Ward C, Lewis S, Coleman T: Prevalence of maternal smoking and environmental tobacco smoke exposure during pregnancy and impact on birth weight: retrospective study using millennium cohort. BMC Publ Health 2007, 7:81.

31. Macdonald-Wallis C, Tobias JH, Davey Smith G, Lawlor DA: Parental smoking during pregnancy and offspring bone mass at age 10 years: findings from a prospective birth cohort. Osteoporos Int 2011, 22:1809-1819.
32. Roddam AW, Pirie K, Pike MC, Chilvers C, Crossley B, Hermon C, McPherson K, Peto J, Vessey M, Beral V: Active and passive smoking and the risk of breast cancer in women aged 36-45 years: a population based case-control study in the UK. Br J Cancer 2007, 97:434-439.

33. Llewellyn DJ, Lang IA, Langa KM, Naughton F, Matthews FE: Exposure to secondhand smoke and cognitive impairment in non-smokers: national cross sectional study with cotinine measurement. BMJ 2009, 338:b462.

34. Williams SA, Kwan SY, Parsons S: Parental smoking practices and caries experience in pre-school children. Caries Res 2000, 34:117-122.

35. MacLennan J, Kafatos G, Neal K, Andrews N, Cameron JC, Roberts R, Evans MR, Cann K, Baxter DN, Maiden MC, Stuart JM: Social behavior and meningococcal carriage in British teenagers. Emerging Infectious Diseases Journal 2006, 12:950-957

36. Coen PG, Tully J, Stuart JM, Ashby D, Viner RM, Booy R: Is it exposure to cigarette smoke or to smokers which increases the risk of meningococcal disease in teenagers? Int J Epidemiol 2006, 35:330-336.

37. Milton B, Cook PA, Dugdill L, Porcellato L, Springett J, Woods SE: Why do primary school children smoke? A longitudinal analysis of predictors of smoking uptake during pre-adolescence. Public Health 2004, 118:247-255.

38. Griesbach D, Amos A, Currie C: Adolescent smoking and family structure in Europe. Soc Sci Med 2003, 56:41-52.

39. Kuschner WG, Reddy S, Mehrotra N, Paintal HS: Electronic cigarettes and thirdhand tobacco smoke: two emerging health care challenges for the primary care provider. International Journal of General Medicine 2011, 4:115-120.

40. Winickoff JP, Friebely J, Tanski SE, Sherrod C, Matt GE, Hovell MF, McMillen RC: Beliefs about the health effects of "thirdhand" smoke and home smoking bans. Pediatrics 2009, 123:e74-e79.

41. Matt GE, Quintana PJ, Zakarian JM, Fortmann AL, Chatfield DA, Hoh E, Uribe AM Hovell MF: When smokers move out and non-smokers move in: residential thirdhand smoke pollution and exposure. Tob Control 2011, 20:e1.

42. Matt GE, Quintana PJE, Hovell MF, Bernert JT, Song S, Novianti N, Juarez T, Floro J, Gehrman C, Garcia M, Larson S: Households contaminated by environmental tobacco smoke: Sources of infant exposures; 2004. [cited 13 (Matt) Department of Psychology, San Diego State University, San Diego, CA 92182-4611, United States]; 1:[29-37].

43. Smoke Outside Campaign. Details available at http://smokeoutside.co.uk.

44. Take 7 Steps Out Campaign. Details available at http://www.take7stepsout. co.uk/.

doi:10.1186/1617-9625-11-3

Cite this article as: Kusel et al:: The impact of smoking in the home on the health outcomes of non-smoker occupants in the UK. Tobacco Induced Diseases 2013 11:3.

\section{Submit your next manuscript to BioMed Central and take full advantage of:}

- Convenient online submission

- Thorough peer review

- No space constraints or color figure charges

- Immediate publication on acceptance

- Inclusion in PubMed, CAS, Scopus and Google Scholar

- Research which is freely available for redistribution 